\title{
Complete Chloroplast Genome Sequence of the Avocado: Gene Organization, Comparative Analysis, and Phylogenetic Relationships with Other Lauraceae
}

\begin{tabular}{|r|l|}
\hline Journal: & Canadian Journal of Forest Research \\
\hline Manuscript ID & cjfr-2016-0199.R1 \\
\hline Manuscript Type: & Article \\
\hline Date Submitted by the Author: & O9-Jul-2016 \\
\hline Complete List of Authors: & $\begin{array}{l}\text { YU, SONG; Xishuangbanna Tropical Botanical Garden; University of the } \\
\text { Chinese Academy of Sciences } \\
\text { YAO, Xin; Xishuangbanna Tropical Botanical Garden; University of the } \\
\text { Chinese Academy of Sciences } \\
\text { TAN, Yunhong; Xishuangbanna Tropical Botanical Garden } \\
\text { GAN, Yi; Zhejiang A \& F University } \\
\text { Corlett, Richard; Xishuangbanna Tropical Botanical Garden }\end{array}$ \\
\hline Keyword: & chloroplast, genome, Persea, Lauraceae, Phylogenetic relationship \\
\hline & \multicolumn{2}{|c}{} \\
\hline
\end{tabular}


2 Complete Chloroplast Genome Sequence of the Avocado: Gene

3 Organization, Comparative Analysis, and Phylogenetic Relationships

4 with Other Lauraceae

5 Yu Song ${ }^{1,2, a}$, Xin Yao ${ }^{1,2, a}$, Yunhong Tan ${ }^{1, \text { a }}$, Yi Gan ${ }^{3}$, Richard T. Corlett $6 \quad 1, *$

$7 \quad{ }^{1}$ Center for Integrative Conservation, Xishuangbanna Tropical Botanical Garden,

8 Chinese Academy of Sciences, Mengla 666303, China

$9 \quad{ }^{2}$ University of Chinese Academy of Sciences, Beijing 100049, China

$10 \quad{ }^{3}$ School of Agriculture and Food Science, Zhejiang A \& F University, Hangzhou 11 311300, China

12 Corresponding author: Richard T. Corlett, Email, corlett@xtbg.org.cn

$13{ }^{a}$ Equal contributors to this work. 


\section{Abstract}

The complete chloroplast sequence of the avocado (Persea americana Mill.), a popular tropical fruit tree, was determined in this study. The plastome consists of $152,723 \mathrm{bp}$, with a typical circular structure including inverted repeats (IRs) 40,104 bp in length separated by a large single-copy region (LSC) and a small single-copy region (SSC) of 93,795 bp and 18,824 bp, respectively. Potential mutation sites including 61 repeats and 679 SSRs were accurately located in the avocado plastome. Compared with the plastomes of five other related Lauraceae, 25 highly variable regions and six micro-inversions were identified and the contraction event of the IR regions was observed. However, the plastomes of Lauraceae are extremely evolutionarily conservative with only $0.32 \%$ sequence divergence, which is surprisingly low for angiosperms. Further phylogenetic analyses were conducted using eight complete plastomes of Lauraceae and seven barcoding sequences from 23 Persea species. These data support a close relationship between the genera Persea and Machilus, and suggest that Persea is polyphyletic.

Keywords: chloroplast; genome; Persea; Lauraceae; Phylogenetic relationship

\section{Introduction}

The genus Persea in the family Lauraceae includes nearly 100 tree species mainly distributed in tropical South America (Rohwer, et al. 2009). The avocado (Persea americana Mill.) was probably domesticated in Mexico and is now widely planted as a popular fruit tree in tropical regions of the world (Chen, et al. 2009). As a member of a basal angiosperm family, the flowers of Persea species have an undifferentiated perianth which represents an intermediate stage before the evolutionary differentiation of sepals and petals (Chanderbali, et al. 2009). However, floral and other morphological traits differ relatively little between Persea species. So, molecular 
methods are needed for species delineation and the resolution of phylogenetic relationships.

In the first molecular study, using the chloroplast marker matK, P. americana, $P$. indica and P. lingue, along with Phoebe formosana, formed a weakly supported clade (the 'Persea group') with Apollonias barbujana, Dehaasia cuneata, and Alseodaphne perakensis (Rohwer 2000). The second, larger, study reported phylogenetic trees for the Lauraceae constructed with different combinations of chloroplast sequences trnL-trnF, psbA-trnH, trnT-trnL, and rpll6 and nuclear barcoding markers 26S rDNA and ITS rDNA, and found a well-supported Persea group including P. americana, $P$. lingue, P. meridensis, and P. caerulea, along with Alseodaphne semecarpifolia, Dehaasia incrassata, Apollonias barbujana, Phoebe formosana and Machilus thunbergii (as P. thunbergii) (Chanderbali, et al. 2001). The intron of $\operatorname{trn} K$ was then used to construct a Bayesian tree with a clade comprising P. americana, P. indica and other species, such as Alseodaphne perakensis, D. cuneata, Apollonias barbujana and M. grijsii (as P. grijsii) (Rohwer and Rudolph 2005).

In order to resolve the relationships among the species in the morphologically defined genus Persea and species placed in related genera, ITS was used in a study including 61 Persea species and 30 other Lauraceae (Rohwer, et al. 2009). The results showed that Persea species were divided between three clades, indicating that they were not a natural group. Another study, using two nuclear markers, ITS and LEAFY intron, confirmed that 'Persea' was polyphyletic (Li, et al. 2011). These studies showed that additional genome sequences and more variable markers are needed to resolve the phylogenetic problems at the lower taxonomic level.

Here, we report the complete chloroplast (cp) genome sequence and the plastomic features of avocado (P. americana), which is the first published complete genome sequence of a member of the genus Persea. The phylogenetic relationships among eight Lauraceae species and 23 Persea species are then reconstructed, suggesting that 
Persea is polyphyletic.

\section{Materials and Methods}

\section{DNA Extraction and Sequencing}

Young leaves of avocado (Persea americana Mill.) (Supplementary Figure S1) were collected from the Xishuangbanna Tropical Botanical Garden $\left(21.9^{\circ} \mathrm{N}, 101.3^{\circ} \mathrm{E} ; 549\right.$ $\mathrm{m}$ above sea level) for genomic DNA extraction using the modified CTAB method (Doyle and Dickson 1987). The whole cp genome was sequenced following Yang et al. (2014), and their nine universal primer pairs were used to perform long-range PCR for next-generation sequencing (Yang, et al. 2014). Following purification, PCR products mixed with six microgram were fragmented into small pieces. According to the manufacturer's manual (Illumina), the cleaved DNA fragments were used to construct 500 bp short-insert libraries. DNA from avovado was indexed by tags and pooled in one lane of a Genome Analyzer (Illumina Hiseq 2500) for sequencing at the Germplasm Bank of Wild Species, Kunming Institute of Botany (KIB) in Kunming, China.

\section{Chloroplast Genome Assembly and Annotation}

Using the cp genome of the related Lauraceae species Machilus balansae (GenBank accession No. KT348517) as a reference (Song, et al. 2015), the Illumina sequence reads were proofread and assembled with trial version of CLC v8 (https://www.qiagenbioinformatics.com). All of the genes encoding proteins, transfer RNAs (tRNA), and ribosomal RNAs (rRNAs) (Figure 1) were annotated on the avocado cp genome using the DOGMA software (Wyman, et al. 2004). The complete avocado cp genome sequence was submitted to GenBank (deposited in GenBank: KX437771).

\section{Repeat Elements, SSRs, and Micro-inversions Detection} REPuter (Kurtz and Schleiermacher 1999) and Tandem Repeats Finder (TRF) programs (Benson 1999) were used to visualize forward, palindrome, reverse, 
complement and tandem sequences in the plastome of avocado, with a minimum repeat size of $18 \mathrm{bp}$ (for REPuter) and $12 \mathrm{bp}$ (for TRF) and a sequence identity greater than $90 \%$. The simple sequence repeats (SSRs) in in the plastome of avocado were identified using Phobos v. 3.3.12 (http://www.ruhr-uni-bochum.de). Repeats were $\geqslant$ $10 \mathrm{bp}$ and three repeat units for mono-, di-, tri-, tetra-, penta-, hexa-, 7-, 8-, 9-, 10-nucleotides. The structures of hairpin loops related to micro-inversions were drawn with RNA structure 5.69

(http://rna.urmc.rochester.edu/RNAstructureWeb/Servers/Predict2/Predict2.html)

\section{Sliding Window Analysis of the Plastomes}

MAFFT version 7 software was used to align the six plastome sequences of avocado (deposited in GenBank: KX437771), Phoebe omeiensis (accession No. KX437772), P. sheareri (accession No. KX437773), Machilus balansae (accession No. KT348517), M. yunnanensis (accession No. KT348516), and Cinnamomum micranthum (accession No. KR014245) (Katoh and Standley 2013). After manual adjustment with BioEdit software http://www.mbio.ncsu.edu/bioedit/bioedit.html), we further conducted a sliding window analysis to calculate the nucleotide variability values (Pi) and the pairwise nucleotide divergence values all over the plastomes in DnaSP version 5 (Librado and Rozas 2009). We defined the window length as 600 base pairs and the step size as 200 base pairs. The values were plotted in the plastomes using an $\mathrm{R}$ program.

\section{Phylogenetic Analyses}

The plastome sequences of Phoebe omeiensis (accession No. KX437772), P. sheareri (accession No. KX437773), Machilus balansae (accession No. KT348517), M. yunnanensis (accession No. KT348516), Cinnamomum micranthum (accession No. KR014245), Endiandra discolor (accession No. KT588615), E. globosa (accession No. KT588614) and Calycanthus fertilis (accession No. NC004993) were collected from GenBank of NCBI. After alignment using MAFFT software with the plastome sequences of avocado, the nine sequences were manually adjusted with BioEdit 
software. The nucleotide substitution model was calculated by the jModeltest 2.0 program and the optimal model of "GTR $+\mathrm{I}+\mathrm{G}$ " was selected (Darriba, et al. 2012). Phylogenetic relationships were reconstructed using a maximum-likelihood (ML) method in MEGA version 5.0 program (Tamura, et al. 2011). One thousand bootstrap replicates were performed in each analysis to obtain the confidence support. The plastome of C. micranthum was used as an out-group.

The DNA sequences of $r b c L$, matK, trnL-trnF, $p s b A-t r n H, n d h F, I T S$, and the intron of $L E A F Y$ of Persea species and Apollonias barbujana were collected from GenBank of NCBI (Supplementary Table S1). All of these sequences were proofread and aligned with MAFFT software. Incongruous sequences of the same species were deleted. The seven sequence matrixes were then manually adjusted and the same sequences with different accession numbers were merged with Sequencher 4.10 (http://www.genecodes.com). The joint matrix including the sequences of $r b c L, m a t K$, trnL-trnF, psbA-trnH, $n d h F, I T S$, and the intron of $L E A F Y$ was constructed with Sequence Matrix 1.7.8 program (Vaidya, et al. 2011), and then used to calculate the nucleotide substitution model with the jModeltest 2.0 program and the optimal model of "GTR $+\mathrm{I}+\mathrm{G}$ " was selected. Phylogenetic relationships were reconstructed using Bayesian analysis in MrBayes 3.1.2 (Ronquist and Huelsenbeck 2003), and the combined analysis was run for 1,000,000 generations, and a burn-in of $25 \%$ was used for the analysis.

\section{Results}

\section{General features of the avocado chloroplast genome}

The plastome of avocado (deposited in Genbank: KX437771), with a length of 152,723 bp, was $132 \mathrm{bp}$ and $153 \mathrm{bp}$ smaller than that of Phoebe omeiensis $(152,855$ bp, GenBank accession No. KX437772) and Phoebe sheareri (152,876 bp, GenBank accession No. KX437773), and 2 bp, 23 bp and 101 bp larger than that of the Machilus balansae (152,721 bp, GenBank accession No. KT348517), Cinnamomum micranthum (152,700 bp, GenBank accession No. KR014245), and M. yunnanensis 
(152,622 bp, GenBank accession No. KT348516), respectively (Song, et al. 2015; Wu, et al. 2015). The $\mathrm{A}+\mathrm{T}$ contents of the six plastomes are all $61 \%$, the lengths of the inverted repeats (IRs), small single-copy region (SSC) and large single-copy region (LSC) in the plastome of avocado are different from the other five species, and were 20,052 bp, 18,824 bp, and 93,795 bp, respectively (Figure 1). The plastome of avocado consists of 79 unique protein-coding genes, 30 transfer RNA (tRNA) genes, and four different ribosome (rRNA) genes. Among all 79 protein coding genes, ten genes, including atpF, petB, petD, $n d h A, n d h B, r p o C 1, r p s 12, r p s 16, r p l 2$, and rpl16, contain one intron, and two genes, $y c f 3$ and $c l p P$, contain two introns (Table 1). There are 15 genes duplicated in the IR regions and 1,374 bp of $3{ }^{\circ}-y c f 1$ are truncated at the IRs' boundaries (Figure 1).

\section{Repeat element and SSR Analysis}

As potential trace of genome rearrangement, repeat motifs play an important role in inducing mutation including substitutions and indels (Cavalier-Smith 2002; Yi, et al. 2013). In the plastome of avocado, 66 repeats, not including the IR region, were detected with REPuter and Tandem Repeats Finder programs. These repeats include 17 forward repeats of $18-33 \mathrm{bp}, 16$ palindromic repeats of $18-48 \mathrm{bp}, 14$ reverse repeats of 18-25 bp, 12 tandem repeats of 12-24 bp and two complement repeats of 18 and 20 bp (Table 2). Most of these repeats (68.9\%) were distributed in the LSC region, whereas $18.0 \%$ were in the IR regions, and $13.1 \%$ in the SSC region. Short tandem repeats, also called simple sequence repeats (SSR), were further identified using the Phobos version 3.3.12 program. A total of 679 potential SSRs were detected in the plastome of avocado, of which 226 are mononucleotides, 38 are dinucleotides, 70 are trinucleotides, 84 are tetranucleotides, 83 are pentanucleotides, 134 are hexanucleotides, 25 are 7-nucleotides, 11 are 8-nucleotides, 6 are 9-nucleotides, and 2 are 10-nucleotides (Figure 2). Most of these potential SSRs (66.0\%) were distributed in the LSC region, whereas $19.4 \%$ were in the IR regions, and $14.6 \%$ in the SSC region (Supplementary Table S2). All of these SSR loci are potentially useful in population studies of Persea species. 


\section{Comparative analysis of the avocado chloroplast genome}

To investigate levels of sequence divergence, we calculated the genetic divergence among the plastomes of avocado, Phoebe omeiensis, Phoebe sheareri, M. balansae, M. yunnanensis and C. micranthum with DnaSP 5.0 software. The nucleotide variability values within $600 \mathrm{bp}$ in the six plastomes varied from 0 to 0.02111 with a mean of 0.00322 . The pairwise nucleotide divergence values between two of the six plastomes varied from 0.001 to 0.005 (Table 3). Both results indicate high sequence similarity across the six plastomes, suggesting that the chloroplast genomes of Lauraceae species are highly conserved. The whole aligned sequences reveal surprising low divergences with only 3 regions displaying high variation. The three most dissimilar regions of the five plastomes were $n d h F-r p l 32$, rpl32-trnL and $y c f 1$ (Figure 3). $n d h F-r p l 32$ and $r p l 32-t r n L$ are located in the SSC region, while $y c f 1$ is in the IR region. All three loci with nucleotide variability more than 0.015 have been reported before as highly variable regions in seed plants (Dong, et al. 2012; Dong, et al. 2015; Korotkova, et al. 2014; Sarkinen and George 2013; Song, et al. 2015). In addition, another 22 loci with nucleotide variability values more than 0.008 were located (Figure 3).

\section{Micro-inversion mutations}

Micro-inversions, forming stable single-stranded hairpin structures, often entail gene duplications and genome rearrangement that can lead to genetic novelty and adaptation (Calvete, et al. 2012; Catalano, et al. 2009). Among the six plastomes of Lauraceae species, we manually identified six micro-inversion events in the regions of $n d h A$ intron, $c c s A-n d h D$, petA-psbJ, rps4-trnT, rps16-trn $Q$ and $p s b A$-trnH (Figure 4A-F). The micro-inversion in the $\operatorname{css} A-n d h D$ intergenic region has been reported before in two Machilus species while three micro-inversions in the rps4-trnT, $y c f 4-c e m A$ and $\operatorname{css} A-n d h D$ intergenic regions have been reported before in two Phoebe species (Song, et al. 2015). In Persea species, two additional micro-inversions with five and 18 bases were detected in the $p s b A$-trnH region (Figure $\mathbf{4 E}$ and $\mathbf{4 G}$ ). 
The loop lengths of these seven micro-inversions varied from $4 \mathrm{bp}$ to $16 \mathrm{bp}$ while their stem length ranged from $9 \mathrm{bp}$ to $25 \mathrm{bp}$. Further comparison of the stem sequences indicated that they are actually short palindromic repeats in the flanks of the micro-inversions (Figure 4).

\section{Phylogeny of Sequenced Lauraceae Plastomes}

To determine the phylogenetic location of avocado with respect to the other Lauraceae with fully sequenced chloroplast genomes, the complete avocado plastome was used to reconstruct the phylogenetic relationships. With the plastome of Calycanthus fertilis (Calycanthaceae, Laurales; GenBank accession No. NC004993) as an outgroup, nine chloroplast genome sequences of Lauraceae, including avocado, Phoebe sheareri, Phoebe omeiensis, M. balansae, M. yunnanensis, C. micranthum, Endiandra discolor (accession No. KT588615), and E. globosa (accession No. KT588614), were aligned by the MAFFT program. A maximum likelihood analysis based on the GTR $+\mathrm{I}+\mathrm{G}$ model was performed with MEGA 5.0 using 1000 bootstrap replicates (Figure 5). The phylogenetic tree reveals that avocado is most closely related to Machilus and Phoebe, which is in agreement with previous reports on the relationships among the three genera (Li, et al. 2011; Rohwer, et al. 2009).

\section{Phylogenetic Analysis of the Persea species}

To determine the phylogenetic relationship of avocado with the other Persea species with reported barcoding data, the available sequences, including $r b c L$, matK, trnL-trnF, psbA-trnH, ndhF, ITS, and intron of $L E A F Y$, were collected

(Supplementary Table S1). The homologous sequences of Apollonias barbujana, $M$. yunnanensis, Phoebe sheareri, and C. micranthum were also collected from NCBI and used as out group. A total of 26 samples including 3 outgroup species were including in the analysis of the data matrix combining nucleotide sequences from the seven cpDNA and nuclear markers. The results of the Bayesian analysis showed that the Persea species can be divided into two clades, indicating that Persea is paraphyletic: a clade including $P$. americana, $P$. floccosa, $P$. gigantea, $P$. nubigena, $P$. 
steyermarkii, P. tolimanensis, P. parvifolia, P. schiedeana and Apollonias barbujana, and a clade comprising $P$. longipes, $P$. lingue, $P$. chamissonis, $P$. caerulea, $P$. indica, $P$. palustris, $P$. haenkeana, $P$. humilis, $P$. borbonia, $P$. major, $P$. meridensis, $P$. weberbaueri, P. splendens, P. aurata and P. alba (Figure 6). In clade I, the avocado, together with another seven Persea species considered as the possible ancestors of avocado, is sister to Apollonias barbujana. The clade consisting of all the species of Persea and Apollonias forms a sister group to M. yunnanensis.

\section{Discussion}

\section{The contraction of the IR region}

This study revealed the whole chloroplast genome for an important tropical fruit crop: avocado of Persea genus in the family Lauraceae. The plastome of avocado with the length of 152,723 bp was smaller than the published plastomes of genus Phoebe and larger than those of Machilus (Song et al., 2015). However, the length of the IR region with 20,052 bp was the smallest among the published plastomes of the Lauraceae. In the published plastomes, the boundaries between IR regions and LSC or SSC are located in two gene sequences, $y c f 1$ and $y c f 2$, which showed incomplete duplication. Unlike $y c f 2$, the lengths of the truncated $y c f 1$ genes were different among the avocado, Phoebe sheareri, Phoebe omeiensis, M. balansae, M. yunnanensis, and C. micranthum. The length of the truncated $y c f 1$ gene with $1377 \mathrm{bp}$ was also the smallest among published plastomes of the Lauraceae. The gene $y c f l$ crossed the $\mathrm{SSC} / \mathrm{IRb}$ region, and the truncated $y c f 1$ gene was located in the IRa region. The length changes in the truncated $y c f 1$ gene directly drave the contraction of the IR regions in the plastome of avocado.

\section{Low sequence divergence among Lauraceae}

Although the length of the truncated $y c f 1$ gene varied and the divergence of the unbroken $y c f l$ gene was high among the avocado, Phoebe sheareri, Phoebe omeiensis, M. balansae, M. yunnanensis, and C. micranthum, the nucleotide variability values of 
the whole plastomes among the species from the four genera were only $0.32 \%$, which approximates the nucleotide variability of two Panax species (0.40\%) (Dong, et al. 2014), three Veroniceae species (0.4\%) (Choi, et al. 2016), and nine Gossypium species $(0.62 \%)(\mathrm{Xu}$, et al. 2012) and was much lower than the sequence divergence among five Epimedium species (3.97\%) (Zhang, et al. 2016) and six Cymbidium species (3.70\%) (Yang, et al. 2013). At the molecular level, the reported chloroplast matK, trnK intron, trnL-trnF, psbA-trnH, trnT-trnL and rpl16 and nuclear ITS and $L E A F$ intron II sequences only partially resolved the phylogenetic and species identification problems in the family Lauraceae (Li et al., 2011). As Rohwer first reported, the genetic divergence within the family is surprisingly low (Rohwer 2000).

\section{Repeat, micro-inversion, and rearrangement mutations}

Although genetic divergence is low overall, there are dozens of divergence hotspots and various mutants, including micro-inversions, were detected in the plastome sequences of species in different genera or the same genus (Figure 4) in Lauraceae. Among the plastomes of avocado, Phoebe sheareri, Phoebe omeiensis, M. balansae, M. yunnanensis, and C. micranthum, six micro-inversion events in the regions of the intron of $n d h A, c c s A-n d h D$, petA-psbJ, rps4-trnT, rps16-trnQ and psbA-trnH were identified. Among 23 species of Persea, two micro-inversion events in the region of $p s b A$-trn $H$ were detected. In the flanks of all these seven micro-inversions, palindromic repeats in pairs with lengths of $9 \mathrm{bp}$ to $25 \mathrm{bp}$ were identified. At the single-stranded hairpin formed in the genome rearrangement process, micro-inversion and palindromic repeat sequences constitute the loop and stem. The affinities of the palindromic repeats and micro-inversions in the plastomes of Lauraceae support the suggestion that repeats are crucial in inducing mutants (Yi, et al. 2013).

\section{Phylogeny of Persea species and sequenced Lauraceae plastomes}

Based on two variable regions from nuclear ITS and $L E A F Y$ intron II, previous molecular phylogenetic analyses indicated that the genus Persea is polyphyletic, which further supported by our phylogenetic analysis of the matrix of five chloroplast 
regions and two nuclear sequences, implying Persea is polyphyletic and there are close relationships between avocado and $P$. floccosa, $P$. gigantea, $P$. nubigena, $P$. steyermarkii, . tolimanensis, $P$. parvifolia, $P$. schiedeana. With species from five genera of Lauraceae, our phylogenomic analysis based on published chloroplast genomes supported a monophyletic Persea group comprising species of Phoebe, Machilus, and Persea genera. Species of Endiandra formed the basal group in the phylogeny, and Cinnamomum is separate from both the Endiandra group and the Persea group as in previously published phylogenetic trees (Chanderbali, et al. 2001; Rohwer 2000; Rohwer and Rudolph 2005).

\section{Acknowledgements}

The authors would like to acknowledge Jun-bo Yang, Jing Yang, Juan-hong Zhang, Chun-yan Lin and Ji-xiong Yang at Kunming Institute of Botany, Chinese Academy Sciences for sequencing technology. This work was supported by the 1000 Talents Program (WQ20110491035). We sincerely thank three anonymous referees for their critical and invaluable comments that greatly improved our manuscript.

\section{Declaration of interest}

The authors report no conflicts of interest. The authors alone are responsible for the content and writing of this article.

\section{References}

Benson, G. 1999. Tandem repeats finder: a program to analyze DNA sequences. Nucleic Acids Res. 27(2): 573-580. doi: Doi 10.1093/Nar/27.2.573.

Calvete, O., Gonzalez, J., Betran, E., and Ruiz, A. 2012. Segmental duplication, microinversion, and gene loss associated with a complex inversion breakpoint region in Drosophila. Mol. Biol. Evol. 29(7): 1875-1889. doi: 10.1093/molbev/mss067.

Catalano, S.A., Saidman, B.O., and Vilardi, J.C. 2009. Evolution of small inversions in chloroplast genome: a case study from a recurrent inversion in angiosperms. Cladistics 25(1): 93-104. doi: 10.1111/j.1096-0031.2008.00236.x. Cavalier-Smith, T. 2002. Chloroplast evolution: Secondary symbiogenesis and multiple losses. Curr. Biol. 12(2): R62-R64. doi: Doi 10.1016/S0960-9822(01)00675-3.

Chanderbali, A.S., Albert, V.A., Leebens-Mack, J., Altman, N.S., Soltis, D.E., and Soltis, P.S. 2009. 
Transcriptional signatures of ancient floral developmental genetics in avocado (Persea americana; Lauraceae). Proc Natl Acad Sci U S A 106(22): 8929-8934. doi: 10.1073/pnas.0811476106. Chanderbali, A.S., van der Werff, H., and Renner, S.S. 2001. Phylogeny and historical biogeography of Lauraceae: Evidence from the chloroplast and nuclear genomes. Ann Mo Bot Gard. 88(1): 104-134. doi: $10.2307 / 2666133$.

Chen, H.F., Morrell, P.L., Ashworth, V.E.T.M., de la Cruz, M., and Clegg, M.T. 2009. Tracing the geographic origins of major avocado cultivars. J. Hered. 100(1): 56-65. doi: 10.1093/jhered/esn068. Choi, K.S., Chung, M.G., and Park, S. 2016. The complete chloroplast genome sequences of three Veroniceae species (Plantaginaceae): comparative analysis and highly divergent regions. Frontiers in Plant Science 7. doi: Artn 355 10.3359/Fpls.2016.00355.

Darriba, D., Taboada, G.L., Doallo, R., and Posada, D. 2012. jModelTest 2: more models, new heuristics and parallel computing. Nat. Methods 9(8): 772. doi: 10.1038/nmeth.2109.

Dong, W., Liu, H., Xu, C., Zuo, Y., Chen, Z., and Zhou, S. 2014. A chloroplast genomic strategy for designing taxon specific DNA mini-barcodes: a case study on ginsengs. BMC Genet 15(1): 138. doi: 10.1186/s12863-014-0138-z.

Dong, W., Liu, J., Yu, J., Wang, L., and Zhou, S. 2012. Highly variable chloroplast markers for evaluating plant phylogeny at low taxonomic levels and for DNA barcoding. PLOS ONE 7(4): e35071. doi: 10.1371/journal.pone.0035071.

Dong, W.P., Xu, C., Li, C.H., Sun, J.H., Zuo, Y.J., Shi, S., Cheng, T., Guo, J.J., and Zhou, S.L. 2015. ycf1, the most promising plastid DNA barcode of land plants. Scientific Reports 5. doi: Artn 8348 10.1038/Srep08348.

Doyle, J.J., and Dickson, E.E. 1987. Preservation of plant-samples for DNA restriction endonuclease analysis. Taxon 36(4): 715-722. doi: Doi 10.2307/1221122.

Katoh, K., and Standley, D.M. 2013. MAFFT multiple sequence alignment software version 7: improvements in performance and usability. Mol. Biol. Evol. 30(4): 772-780. doi: 10.1093/molbev/mst010. Korotkova, N., Nauheimer, L., Ter-Voskanyan, H., Allgaier, M., and Borsch, T. 2014. Variability among the most rapidly evolving plastid genomic regions is lineage-specific: implications of pairwise genome comparisons in Pyrus (Rosaceae) and other angiosperms for marker choice. PLOS ONE 9(11). doi: ARTN e112998 10.1371/journal.pone.0112998.

Kurtz, S., and Schleiermacher, C. 1999. REPuter: fast computation of maximal repeats in complete genomes. Bioinformatics 15(5): 426-427. doi: DOI 10.1093/bioinformatics/15.5.426.

Li, L., Li, J., Rohwer, J.G., van der Werff, H., Wang, Z.H., and Li, H.W. 2011. Molecular phylogenetic analysis of the Persea group (Lauraceae) and its biogeographic implications on the evolution of tropical and subtropical Amphi-Pacific disjunctions. Am. J. Bot. 98(9): 1520-1536. doi: 10.3732/ajb.1100006. Librado, P., and Rozas, J. 2009. DnaSP v5: a software for comprehensive analysis of DNA polymorphism data. Bioinformatics 25(11): 1451-1452. doi: 10.1093/bioinformatics/btp187.

Rohwer, J.G. 2000. Toward a phylogenetic classification of the Lauraceae: Evidence from matK sequences. Syst. Bot. 25(1): 60-71. doi: Doi 10.2307/2666673.

Rohwer, J.G., and Rudolph, B. 2005. Jumping genera: the phylogenetic positions of Cassytha, Hypodaphnis, and Neocinnamomum (Lauraceae) based on different analyses of trnK intron sequences. Ann Mo Bot Gard. 92(2): 153-178

Rohwer, J.G., Li, J., Rudolph, B., Schmidt, S.A., van der Werff, H., and Li, H.W. 2009. Is Persea 
(Lauraceae) monophyletic? Evidence from nuclear ribosomal ITS sequences. Taxon 58(4): 1153-1167. Ronquist, F., and Huelsenbeck, J.P. 2003. MrBayes 3: Bayesian phylogenetic inference under mixed models. Bioinformatics 19(12): 1572-1574.

Sarkinen, T., and George, M. 2013. Predicting plastid marker variation: can complete plastid genomes from closely related species help? PLOS ONE 8(11): e82266. doi: 10.1371/journal.pone.0082266. Song, Y., Dong, W.P., Liu, B., Xu, C., Yao, X., Gao, J., and Corlett, R.T. 2015. Comparative analysis of complete chloroplast genome sequences of two tropical trees Machilus yunnanensis and Machilus balansae in the family Lauraceae. Frontiers in Plant Science 6. doi: Artn 662 10.3389/Fpls.2015.00662. Tamura, K., Peterson, D., Peterson, N., Stecher, G., Nei, M., and Kumar, S. 2011. MEGA5: molecular evolutionary genetics analysis using maximum likelihood, evolutionary distance, and maximum parsimony methods. Mol. Biol. Evol. 28(10): 2731-2739. doi: 10.1093/molbev/msr121. Vaidya, G., Lohman, D.J., and Meier, R. 2011. SequenceMatrix: concatenation software for the fast assembly of multi-gene datasets with character set and codon information. Cladistics 27(2): 171-180. doi: 10.1111/j.1096-0031.2010.00329.x. Wu, C.C., Ho, C.K., and Chang, S.H. 2015. The complete chloroplast genome of Cinnamomum kanehirae Hayata (Lauraceae). Mitochondrial DNA 27(4): 2681-2682. doi: 10.3109/19401736.2015.1043541.

Wyman, S.K., Jansen, R.K., and Boore, J.L. 2004. Automatic annotation of organellar genomes with DOGMA. Bioinformatics 20(17): 3252-3255. doi: DOI 10.1093/bioinformatics/bth352.

Xu, Q., Xiong, G., Li, P., He, F., Huang, Y., Wang, K., Li, Z., and Hua, J. 2012. Analysis of complete nucleotide sequences of 12 gossypium chloroplast genomes: origin and evolution of allotetraploids. PLOS ONE 7(8): e37128. doi: 10.1371/journal.pone.0037128.

Yang, J.B., Li, D.Z., and Li, H.T. 2014. Highly effective sequencing whole chloroplast genomes of angiosperms by nine novel universal primer pairs. Mol. Ecol. Resour. 14(5): 1024-1031. doi: 10.1111/1755-0998.12251.

Yang, J.B., Tang, M., Li, H.T., Zhang, Z.R., and Li, D.Z. 2013. Complete chloroplast genome of the genus Cymbidium: lights into the species identification, phylogenetic implications and population genetic analyses. BMC Evol. Biol. 13(1): 84. doi: 10.1186/1471-2148-13-84. Yi, X., Gao, L., Wang, B., Su, Y.J., and Wang, T. 2013. The complete chloroplast genome sequence of Cephalotaxus oliveri (Cephalotaxaceae): evolutionary comparison of Cephalotaxus chloroplast DNAs and insights into the loss of inverted repeat copies in gymnosperms. Genome Biol. Evol. 5(4): 688-698. doi: $10.1093 /$ gbe/evt042.

Zhang, Y.J., Du, L.W., Liu, A., Chen, J.J., Wu, L., Hu, W.M., Zhang, W., Kim, K., Lee, S.C., Yang, T.J., and Wang, Y. 2016. The complete chloroplast genome sequences of five epimedium species: lights into phylogenetic and taxonomic analyses. Frontiers in Plant Science 7. doi: Artn 306 10.3389/Fpls.2016.00306.

Table 1. Genes encoded by avocado plastomes

\begin{tabular}{lll}
\hline Category for genes & Group of gene & Name of gene \\
\hline $\begin{array}{l}\text { Photosynthesis related } \\
\text { genes }\end{array}$ & Rubisco & $r b c L$ \\
& Photosystem I & psaA,psaB,psaC,psaI,psaJ \\
& $\begin{array}{l}\text { Assembly/stability of } \\
\text { photosystem I }\end{array}$ & $* * y c f 3, y c f 4$ \\
&
\end{tabular}


Transcription and translation related genes

RNA genes

Other genes

Genes of unknown function

Pseudogenes

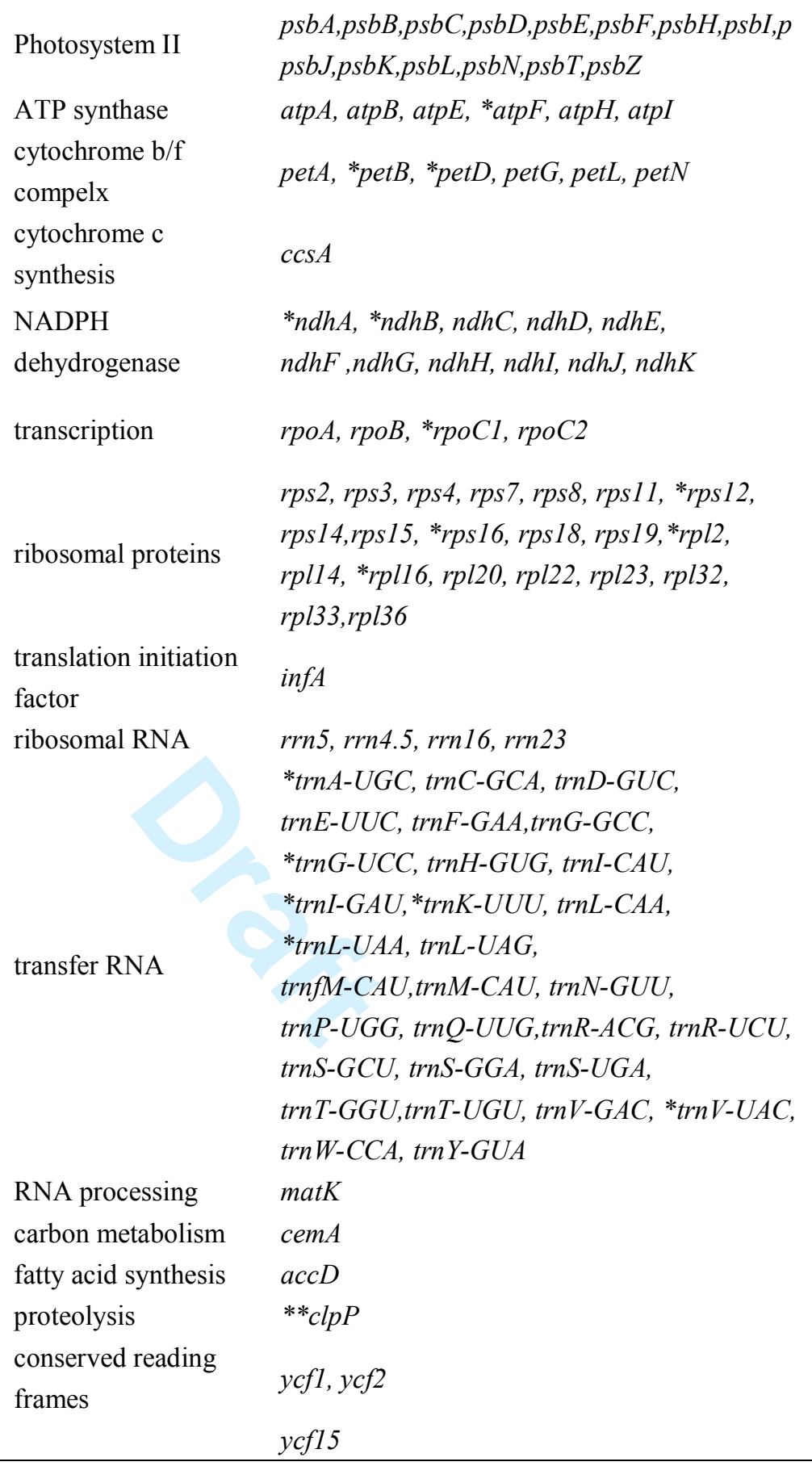

Asterisks before gene names indicate intron containing genes $(*)$, and double asterisks indicate two introns in the gene.

429 Table 2. Locations of repeats in the avocado plastome.

\begin{tabular}{llrllrrrr}
\hline $\begin{array}{l}\text { Repeat A } \\
\text { Location }\end{array}$ & $\begin{array}{c}\text { Start } \\
\text { locus }\end{array}$ & Region & $\begin{array}{l}\text { Repeat B } \\
\text { Location }\end{array}$ & $\begin{array}{l}\text { Start } \\
\text { locus }\end{array}$ & Region & Length & Type \\
\hline 1 & trnH-psbA & 215 & LSC & clpP intron1 & 74263 & LSC & 18 & P \\
2 & trnH-psbA & 215 & LSC & rpl2-rpl23 & 89466 & LSC & 18 & C \\
3 & trnH-psbA & 216 & LSC & $n d h F-r p l 32$ & 116177 & SSC & 18 & F
\end{tabular}




\begin{tabular}{|c|c|c|c|c|c|c|c|}
\hline 4 & $\operatorname{trn} H-p s b A$ & 220 & LSC & rpsl6 intron & 5597 & LSC & 18 \\
\hline 5 & $\operatorname{trn} H-p s b A$ & 220 & LSC & atpA-atpF & 13004 & LSC & 18 \\
\hline 6 & trnH-psbA & 222 & LSC & $\operatorname{trn} G-U C C$ & 10963 & LSC & 18 \\
\hline 7 & $\operatorname{trn} K-U U U$ & 1892 & LSC & rpoB-trnC & 28529 & LSC & 20 \\
\hline 8 & $\operatorname{trnK-rps16}$ & 4702 & LSC & $\operatorname{trnK-rps16}$ & 4702 & LSC & 26 \\
\hline 9 & $\operatorname{trnK}$-rps 17 & 5180 & LSC & psbM-trnD & 31914 & LSC & 18 \\
\hline 10 & $\operatorname{trnK}$-rps 18 & 5180 & LSC & petA-psbJ & 66643 & LSC & 18 \\
\hline 11 & $\operatorname{trnK}$-rps 19 & 5180 & LSC & rps19-rpl2 & 87884 & LSC & 18 \\
\hline 12 & rps16 intron & 5594 & LSC & rps16 intron & 5594 & LSC & 18 \\
\hline 13 & rps16 & 5596 & LSC & atpA-atpF & 13004 & LSC & 19 \\
\hline 14 & $\operatorname{trn} S-G C U$ & 9502 & LSC & $\operatorname{trn} S-U G A$ & 37928 & LSC & 18 \\
\hline 15 & rps2-rpoC2 & 17386 & LSC & rps15-ycf1 & 128110 & $\mathrm{SSC}$ & 19 \\
\hline 16 & rpoC2 & 18825 & LSC & rрос2 & 18825 & LSC & 19 \\
\hline 17 & rров & 25027 & LSC & rров & 25027 & LSC & 19 \\
\hline 18 & $p s b M-t r n D$ & 31914 & LSC & petA-psbJ & 66638 & LSC & 23 \\
\hline 19 & $p s b M-t r n D$ & 31914 & LSC & rps19-rpl2 & 87883 & LSC & 19 \\
\hline 20 & $\operatorname{trn} E-\operatorname{trn} T$ & 33285 & LSC & rps14 & 39396 & LSC & 26 \\
\hline 21 & $\operatorname{trnS}-U G A$ & 37858 & LSC & $\operatorname{trn} S-G G A$ & 47579 & LSC & 21 \\
\hline 22 & $\operatorname{lhbA-trnG}$ & 38669 & LSC & $\operatorname{lhb} A-\operatorname{trn} G$ & 38669 & LSC & 28 \\
\hline 23 & $\operatorname{trn} G-\operatorname{trnf} M$ & 38932 & LSC & $y c f 1$ & 129626 & $\mathrm{SSC}$ & 20 \\
\hline 24 & $\operatorname{trnf} M-C A U$ & 38964 & LSC & $\operatorname{trn} P-U G G$ & 70393 & LSC & 21 \\
\hline 25 & $\operatorname{trn} T$-trnL & 49332 & LSC & $\operatorname{trn} T$-trnL & 49332 & LSC & 19 \\
\hline 26 & $\operatorname{trn} T-\operatorname{trn} L$ & 49335 & LSC & $n d h F-r p l 32$ & 116166 & $\mathrm{SSC}$ & 19 \\
\hline 27 & $n d h C$ & 52463 & LSC & $n d h C$ & 52463 & LSC & 20 \\
\hline 28 & $n d h C-\operatorname{trn} V$ & 53121 & LSC & $n d h C-\operatorname{trn} V$ & 53121 & LSC & 25 \\
\hline 29 & $n d h C-t r n V$ & 53121 & LSC & rps3-rps19 & 87040 & LSC & 19 \\
\hline 30 & $n d h C-t r n V$ & 53127 & LSC & rps3-rps19 & 87038 & LSC & 21 \\
\hline 31 & petA-psbJ & 66642 & LSC & rps19-rpl2 & 87883 & $\mathrm{SSC}$ & 20 \\
\hline 32 & rpl20-rps 12 & 72846 & LSC & clpP intron 2 & 74944 & LSC & 20 \\
\hline 33 & clpP intron 1 & 74263 & LSC & rpl2-rpl23 & 89465 & LSC & 19 \\
\hline 34 & $p s b T-p s b N$ & 77964 & LSC & $p s b T-p s b N$ & 77964 & LSC & 48 \\
\hline 35 & rps19-rpl2 & 87914 & LSC & $y c f 2$ & 93829 & IRa & 22 \\
\hline 36 & rps19-rpl2 & 87914 & LSC & $y c f 2$ & 152667 & $\mathrm{IRb}$ & 22 \\
\hline 37 & rpl2-rpl23 & 89458 & LSC & rpl2-rpl23 & 89458 & LSC & 25 \\
\hline 38 & $y c f 2$ & 95123 & IRa & $y c f 2$ & 95147 & IRa & 33 \\
\hline 39 & $y c f 2$ & 95123 & IRa & $y c f 2$ & 151338 & $\mathrm{IRb}$ & 33 \\
\hline 40 & $y c f 2$ & 95147 & IRa & $y c f 2$ & 151362 & $\mathrm{IRb}$ & 33 \\
\hline 41 & $\operatorname{trn} N-y c f 1$ & 112248 & IRa & $\operatorname{trn} N-y c f 1$ & 112267 & IRa & 21 \\
\hline 42 & $\operatorname{trn} N-y c f 1$ & 112248 & IRa & $\operatorname{trn} N-y c f 1$ & 134230 & $\mathrm{IRb}$ & 21 \\
\hline 43 & $\operatorname{trn} N-y c f 1$ & 112267 & IRa & $\operatorname{trn} N-y c f 1$ & 134249 & $\mathrm{IRb}$ & 21 \\
\hline 44 & $\operatorname{ccs} A-n d h D$ & 119975 & $\mathrm{SSC}$ & $\operatorname{ccs} A-n d h D$ & 120005 & LSC & 25 \\
\hline 45 & ndhA intron & 125655 & $\mathrm{SSC}$ & ndhA intron & 125655 & $\mathrm{SSC}$ & 19 \\
\hline 46 & $y c f 1$ & 129875 & $\mathrm{SSC}$ & $y c f 1$ & 129875 & $\mathrm{SSC}$ & 19 \\
\hline 47 & $y c f 1$ & 132638 & $\mathrm{SSC}$ & $y c f 1$ & 132656 & $\mathrm{IRb}$ & 30 \\
\hline
\end{tabular}




\begin{tabular}{llrllrlll}
48 & $y c f 1-t r n N$ & 134230 & IRb & $y c f 1-t r n N$ & 134249 & IRb & 21 & $\mathrm{~F}$ \\
49 & $y c f 2$ & 151338 & IRb & $y c f 2$ & 151362 & IRb & 33 & $\mathrm{~F}$ \\
50 & trnK intron & 3906 & LSC & trnK intron & 3930 & LSC & 12 & $\mathrm{~T}$ \\
51 & trnE-trnT & 33579 & LSC & trnE-trnT & 33617 & LSC & 15 & $\mathrm{~T}$ \\
52 & trnT-psbD & 34987 & LSC & trnT-psbD & 35019 & LSC & 17 & $\mathrm{~T}$ \\
53 & rps4-trnT & 48574 & LSC & $r p s 4-t r n T$ & 48605 & LSC & 13 & $\mathrm{~T}$ \\
54 & trnT-trnL & 48942 & LSC & trnT-trnL & 48969 & LSC & 13 & $\mathrm{~T}$ \\
55 & trnF-ndhJ & 50724 & LSC & trnF-ndhJ & 50759 & LSC & 18 & $\mathrm{~T}$ \\
56 & accD-psaI & 62178 & LSC & accD-psaI & 62223 & LSC & 15 & $\mathrm{~T}$ \\
57 & psbE-petL & 69155 & LSC & $p s b E-p e t L$ & 69197 & LSC & 21 & $\mathrm{~T}$ \\
58 & rpl2-rpl23 & 89457 & LSC & $r p l 2-r p l 23$ & 89483 & LSC & 13 & $\mathrm{~T}$ \\
59 & rpl2-rpl23 & 89459 & LSC & $r p l 2-r p l 23$ & 89485 & LSC & 14 & $\mathrm{~T}$ \\
60 & $y c f 2$ & 92333 & LSC & $y c f 2$ & 92383 & LSC & 21 & $\mathrm{~T}$ \\
61 & rpl32-trnL & 118212 & SSC & $r p l 32-t r n L$ & 118244 & SSC & 16 & $\mathrm{~T}$ \\
\hline
\end{tabular}

F: forward repeats; P: palindromic repeats; R: reverse repeats; C: complement repeats; T: tandem repeats. 
430 Table 3. Pairwise nucleotide divergences of the six plastomes of Lauraceae.

\begin{tabular}{|c|c|c|c|c|c|c|}
\hline & $\begin{array}{c}\text { Persea } \\
\text { americana }\end{array}$ & $\begin{array}{l}\text { Machilus } \\
\text { balansa }\end{array}$ & $\begin{array}{l}\text { Machilus } \\
\text { yunnanensis }\end{array}$ & $\begin{array}{l}\text { Phoebe } \\
\text { omeiensis }\end{array}$ & $\begin{array}{l}\text { Phoebe } \\
\text { sheareri }\end{array}$ & $\begin{array}{l}\text { Cinnamomum } \\
\text { micranthum }\end{array}$ \\
\hline \multicolumn{7}{|l|}{ Persea } \\
\hline americana & - & 0.0032 & 0.0033 & 0.0028 & 0.0028 & 0.0044 \\
\hline balansa & 0.0032 & - & 0.0015 & 0.0027 & 0.0027 & 0.0049 \\
\hline \multicolumn{7}{|l|}{ Machilus } \\
\hline yunnanensis & 0.0033 & 0.0015 & - & 0.0029 & 0.0029 & 0.0050 \\
\hline omeiensis & 0.0028 & 0.0027 & 0.0029 & - & 0.0010 & 0.0045 \\
\hline \multicolumn{7}{|l|}{ Phoebe } \\
\hline sheareri & 0.0028 & 0.0027 & 0.0029 & 0.0010 & - & 0.0044 \\
\hline \multicolumn{7}{|l|}{ Cinnamomum } \\
\hline micranthum & 0.0044 & 0.0049 & 0.0050 & 0.0045 & 0.0044 & - \\
\hline
\end{tabular}


Figure 1. Gene map of the avocado plastome. The annotation of the genome was performed using DOGMA. The genes that are drawn outside of the circle are transcribed clockwise, while those inside are counterclockwise. Small single copy (SSC), large single copy (LSC), and inverted repeats (IRa, IRb) are indicated.

Figure 2. Number and type of simple sequence repeats of the avocado plastome.

The SSRs was identified using Phobos version 3.3.12 program. SSRs were classified as the number of repeat unit length.

Figure 3. Comparison of the nucleotide variability (Pi) values of the six

plastomes of Lauraceae. The genetic divergence among the plastomes of avocado, Phoebe omeiensis, Phoebe sheareri, Machilus balansae, M. yunnanensis and Cinnamomum micranthum was calculated with DnaSP 5.0 software. (window length: $600 \mathrm{bp}$, step size: 200bp). X-axis: position of the midpoint of a window, Y-axis: nucleotide diversity of each window.

Figure 4. The predicted hairpin loops of inversions in the six plastomes of Lauraceae. The inversions among the plastomes of avocado, Phoebe omeiensis, Phoebe sheareri, Machilus balansae, M. yunnanensis and Cinnamomum micranthum were manual identified. The structures of hairpin loops were drawn with RNAstructure 5.6. The arrows in the figure indicate the break points in inversion events.

\section{Figure 5. Molecular phylogenetic tree of eight species of Lauraceae based on} complete plastome sequences. The phylogenetic tree was constructed from available chloroplast genome sequences of eight species in the family Lauraceae by maximum likelihood (ML) method with bootstrapping analysis (1000 reiterations) in MEGA version 5.0 program. The tree is rooted with the plastome sequence of Calycanthus fertilis. 
463 Figure 6. Phylogenetic tree reconstruction of 18 taxa using Bayesian inference

464 based on concatenated sequences of seven sequences. The confederate matrix

465 including $r b c L$, matK, trnL-trnF, psbA-trnH, $n d h F, I T S$, and intron of LEAFY

466 sequences of Persea species, Apollonias barbujana, Machilus yunnanensis, Phoebe

467 sheareri and Cinnamomum micranthum was used to reconstruct the phylogenetic

468 relationship of Persea species using Bayesian Inference (BI) method in MrBayes

469 3.1.2. The combined analysis was run for $1,000,000$ generations, and a burn-in of $25 \%$

470 was used for the analysis. The tree is rooted with the sequences of Phoebe sheareri.

471

472

\section{Supplementary Material}

474 Figure $\mathbf{S 1}$. The fruiting branches of avocado.

475 Table S1. Genbank accession numbers for DNA sequences of $r b c L$, matK, $\operatorname{trnL}$-trnF, 476 psbA-trnH, ndhF, ITS, and intron of LEAFY spquences of Persea species, Apollonias

477 barbujana, Machilus yunnanensis, Phoebe sheareri and Cinnamomum micranthum.

478 Table S2. Distribution of simple sequence repeats in the avocado plastome. 